\title{
On the Negativity of Moduli Spaces for Polarized Manifolds
}

\author{
Kang Zuo ${ }^{1}$
}

Received: 21 May 2020 / Accepted: 8 April 2021 / Published online: 5 June 2021

(C) The Author(s) 2021

\begin{abstract}
Given a log base space $(Y, S)$, parameterizing a smooth family of complex projective varieties with semi-ample canonical line bundle, we briefly recall the construction of the deformation Higgs sheaf and the comparison map on $(Y, S)$ made in the work by ViehwegZuo. While almost all hyperbolicities in the sense of complex analysis such as Brody, Kobayashi, big Picard and Viehweg hyperbolicities of the base $U=Y \backslash S$ (under some technical assumptions) follow from the negativity of the kernel of the deformation Higgs bundle we pose a conjecture on the topological hyperbolicity on $U$. In order to study the rigidity problem we then introduce the notions of the length and characteristic varieties of a family $f: X \rightarrow Y$, which provide an infinitesimal characterization of products of sub $\log$ pairs in $(Y, S)$ and an upper bound for the number of subvarieties appearing as factors in such a product. We formulate a conjecture on a characterization of non-rigid families of canonically polarized varieties.
\end{abstract}

Keywords Moduli space $\cdot$ Hyperbolicity

Mathematics Subject Classification (2010) $14 \mathrm{~J} 10 \cdot 32 \mathrm{G} 13 \cdot 32 \mathrm{C} 18$

\section{Introduction}

Let $U$ be a complex quasi-projective manifold and $\bar{Y}=U \cup \bar{S}$ a smooth compactification. Hodge theory plays a fundamental role in studying the geometry of $U$. We are particularly interested on $U$ as a base space $f: V \rightarrow U$ parameterizing smooth projective varieties. The powerful theory on variation of Hodge structures developed by P. Griffiths [11-13] suggests certain negativity of the sheaf of logarithmic holomorphic vector fields of the log pair $(\bar{Y}, \bar{S})$. We detail here the resulting program of the complex hyperbolicity of $U$ in its various aspects as well as the program motivated by the original Shafarevich conjecture.

Dedicated to Professor Jürgen Jost on the occasion of his 65th birthday.

Kang Zuo

zuok@uni-mainz.de

1 Institut fur Mathematik, Universität Mainz, Mainz, 55099, Germany 
In Section 2, we summarize all these relevant properties for a base admitting a locally injective Torelli map. C. Simpson [25] has introduced a basic notion in nonabelian Hodge theory, the so-called graded Higgs bundle or the system of Hodge bundles $(E, \theta)$ as the grading of a polarized variation of Hodge structures (PVHS). These $\mathcal{O}_{Y}$-linear objects are better understandable in algebraic geometry and enjoy a crucial property in complex geometry: The kernel of the Kodaira-Spencer map (the Higgs field) $\operatorname{ker}(\theta)$ is semi-negative.

In Section 3, we discuss the work by Viehweg-Zuo on families without the assumption of local injective Torelli maps. We emphasize the following crucial new ingredients in Viehweg-Zuo's construction:

- The deformation Higgs bundle arising from the Kodaira-Spencer theory. One notes that if the family has maximal variation then the Kodaira-Spencer map is generically injective.

- Constructing a comparison map between the deformation Higgs bundle and a Higgs bundle of geometric origin.

With the help of this comparison map, the negativity of the kernel of the Higgs field mentioned in Section 2 is transformed into that of the kernel of Kodaira-Spencer map on the deformation Higgs bundle. However we do not achieve all known negativity properties which are enjoyed by families admitting locally injective Torelli map. We pose several questions and conjectures. In particular, we raise a conjecture on the topological hyperbolicity of the base space, which is motivated by a question asked by Jürgen Jost some years ago on the negativity of Riemannian sectional curvature of a base space admitting a locally injective Torelli map.

In Section 4 we consider the rigidity problem of families, or equivalently products of subvarieties of the base space. The original Shafarevich program asked for the so-called finiteness of the set $\mathcal{H}$ of isomorphic classes of families over a fixed log base. The finiteness is decomposed into two basic problems:

- $\quad$ The boundedness of $\mathcal{H}$.

- The rigidity of points in $\mathcal{H}$.

The boundedness has been proven by various people. For example, for semi-stable families of abelian varieties over a base curve Faltings [9] has shown the boundedness. Jost and Yau [16] have used Yau's form of Schwarz inequality and given another proof of the boundedness for families of abelian varieties over curves. In general, Viehweg and Zuo [29] have shown the boundedness for families of varieties with semi-ample canonical line bundles over a base curve without requiring the existence of a locally injective Torelli map. Finally, Kovács, Lieblich [18] have shown the boundedness for families over a higher dimensional base.

The rigidity problem is subtle. In fact, there exists non-rigid families of higher dimensional varieties. So characterizing non-rigid families will be important for Shafarevich program for families of higher dimension varieties. In Section 4.1 we introduce the notion of the length of a family, which generalizes the rank of a bounded symmetric domain. In Section 4.2 we define the characteristic varieties of a family, which gives an infinitesimal characterization of products of subvarieties of the base space. The characteristic varieties has been introduced by N. Mok originally for bounded symmetric domain for studying the metric rigidity problems. Motivated by Mok's work, M. Sheng and the author have constructed characteristic varieties for PVHS of Calabi-Yau type. With help of such new invariants we hope to get a better understanding of the Arakelov rigidity problem for families of higher dimensional varieties. 
Very recently, Javanpeykar, Sun and Zuo [15] proved a new version of the Shafarevich conjecture, the so-called finiteness of pointed families $f: X \rightarrow Y$ of $h$-dimensional polarized varieties. A $\log$ map from a $\log$ curve $\phi:\left(C, S_{C}\right) \rightarrow(Y, S)$ has only the trivial deformation $\phi_{t}$ if $\phi_{t}$ fixes no less than $\frac{1}{2}(h-1) \operatorname{deg} \Omega_{C}^{1}\left(\log S_{C}\right)$ number of points in $C \backslash S_{C}$. We remark that the slop of the ample vector bundle $f_{*} \omega_{X / C}^{v}$ satisfies the following Arakelov inequality

$$
\mu f_{*} \omega_{X / C}^{v} \leq \frac{h v}{2} \operatorname{deg} \Omega_{C}^{1}\left(\log S_{C}\right)
$$

\section{Semi-negativity of the Kernel of Logarithmic Higgs Field and Bigness of Logarithmic Differential Forms on Base Space Admitting Locally Injective Torelli Map}

Let $(\bar{Y}, \bar{S})$ be a smooth $\log$ pair and $\left(V, \nabla, \mathrm{Fil}^{*}, \Psi\right)$ a polarized variation of complex Hodge structures on $U:=\bar{Y} \backslash \bar{S}$ with quasi-unipotent local monodromy around $\bar{S}$. Let $(E, \theta)$ be the grading of the quasi-canonically extended filtered de Rham bundle, which is originally observed by C. Simpson in non-abelian Hodge theory. It is a graded logarithmic Higgs bundle, or a system of logarithmic Hodge bundles called by Simpson. Comparing with the connections on the de Rham bundle, the Higgs field $\theta$ is $\mathcal{O}_{Y}$-linear and seems to be better understood in algebraic geometry and complex geometry. Let $K \subset E$ be the kernel of the Higgs map $\theta: E \rightarrow E \otimes \Omega_{\bar{Y}}(\log \bar{S})$. Based on the Griffiths curvature formula for the Hodge metric on $E$ and the asymptotic behavior of the Hodge metric on the quasi-canonical extension on $\bar{S}$ due to Cattani-Kaplan-Schmid [3], one shows that the curvature current of the Hodge metric on $K$ is semi negative. In the same way, using the polarized variation of mixed Hodge structures along $S$, one shows (in [32]) that $K$ is also semi-negative along $\bar{S}$.

\subsection{Semi-negativity of $T_{\bar{\gamma}}(-\log \bar{S})$}

The most important negativity is that of the logarithmic tangent bundle $T_{\bar{Y}}(-\log \bar{S})$. Assuming injectivity of the local Torelli, i.e., the injectivity of the derivative of the period $\operatorname{map} \phi$

$$
d \phi: T_{\bar{Y}}(-\log \bar{S}) \rightarrow \operatorname{End}(E)
$$

on $U$ (in general $d \phi$ is required to be injective at at least one point in $U$ ), since the original Higgs map satisfies the integrability condition: $\theta \wedge \theta=0$, this is equivalent to ([32, Proposition 2.1])

$$
d \phi\left(T_{\bar{Y}}(-\log \bar{S})\right) \subset \operatorname{Ker}\left(\theta^{\text {end }}: \operatorname{End}(E) \rightarrow \operatorname{End}(E) \otimes \Omega_{\bar{Y}}^{1}(\log \bar{S})\right),
$$

where the Higgs map $\theta^{\text {end }}$ comes from the tensor algebra and gives also the grading of the Gauss-Manin connection on $\operatorname{End}\left(V, \nabla, \mathrm{Fil}^{*}, \Psi\right)$. In particular, $T_{\bar{Y}}(-\log \bar{S})$ is seminegative in the sense of the curvature current of the Hodge metric. Consequently, it implies that

- $\Omega_{\bar{Y}}(\log \bar{S})$ is weakly positive on $U$ in the sense of Viehweg.

- $\Omega_{\bar{Y}}(\log \bar{S})$ is semi-positive in the usual sense of Algebraic Geometry, i.e., for any projective curve $\bar{C} \subset \bar{Y}$ and any quotient bundle $\left.\Omega_{\bar{Y}}(\log \bar{S})\right|_{\bar{C}} \rightarrow Q \rightarrow 0$ one has $\operatorname{det} Q \geq 0$. 
- The holomorphic bisectional curvature of the Hodge metric on $\left.T_{\bar{Y}}(-\log \bar{S})\right|_{U}$ is seminegative.

\subsection{Bigness of $\omega_{\bar{Y}}(\bar{S})$}

The theorem due to Griffiths-Schmid [14] tells us more! It asserts that the holomorphic sectional curvature is strictly negative. This property together with semi-negativity of $T_{\bar{Y}}(-\log \bar{S})$ shows that $\omega_{\bar{Y}}(\bar{S})$ is big. Griffiths $[10,13]$ constructed the so-called augmented line bundle $L$ by taking the product of determinants of the Hodge bundles of suitable powers. $L$ is nef on $Y$ and ample on $U$. Alternatively, one uses the iteration of the Kodaira-Spencer map and shows directly $L=r \omega_{\bar{Y}}(\bar{S})-P+N$, where $r$ is a positive rational number, $P$ is an effective divisor and $N$ is a rational semi-negative divisor. This shows $\omega_{Y}(S)$ is big ([32, Lemma 2.2]).

\subsection{Bigness of $\Omega_{\bar{Y}}^{1}(\log \bar{S})$}

The bigness of $\Omega_{\bar{Y}}(\log \bar{S})$ should have a close relation to the strict negativity of holomorphic sectional curvature due to Griffiths-Schmid. There are several different notions of ampleness and bigness for torsion free sheaves on $Y$. The first one has been introduced by Viehweg and mainly used in Viehweg-Zuo's papers. Here we just concentrate on vector bundles.

Definition 1 (Viehweg) A vector bundle $E$ over a projective variety is called generically ample if for some symmetric power $S^{n}(E)$ there exist an ample line bundle $A$ and a generically surjective map

$$
A^{\oplus m} \rightarrow S^{n}(E)
$$

Definition 2 A vector bundle $E$ is called big if some positive power of the tautological line bundle $O(1)$ on the projective bundle $\mathbb{P}(E)$ contains an ample sub-line bundle.

Proposition 1 1. E is big in Definition 2 if and only if some symmetric power $S^{n}(E)$ contains a generically ample subbundle.

2. A generically ample vector bundle is big.

Conversely a big vector bundle is not necessarily generically ample. For example, the cotangent bundle on a locally bounded symmetric domain of rank $>1$ is big but not generically ample. However, the cotangent bundle on a complex ball quotient is ample.

In general, the sheaf of log differential forms on a log base $(\bar{Y}, \bar{S})$ admitting a locally injective Torelli map is big. This is due to Brunebarbe, Klingler and Totaro ([2, Theorem 1.1]). The crucial point in Lemma 1.4 in their paper is to use the strict negativity of holomorphic sectional curvature to produce a generically ample subsheaf in a symmetric power $S^{n}\left(\Omega_{\bar{Y}}^{1}(\log \bar{S})\right)$. In fact, the existence of an ample subsheaf in the symmetric power of sheaf of log differential forms can be constructed directly via Kodaira-Spencer map as follows. If the first Hodge bundle $E^{n, 0}$ is generically ample as in Definition 1, for example in the case of variation of middle cohomology of Calabi-Yau $n$-folds or abelian varieties, then one obtains a generically ample subbundle

$$
E^{n, 0} \otimes \operatorname{ker}(\theta)^{\vee} \rightarrow S^{m}\left(\Omega_{\bar{Y}}^{1}(\log \bar{S})\right)
$$

by applying the maximal non-zero iteration of Kodaira-Spencer maps. In general we consider the Griffiths augmented line bundle $L$. Then $L$ is generically ample. Deng [6] observed 
that $L$ can be realized as a sub-line bundle in a suitable type of tensor product of the original Higgs bundle. By running the maximal non-zero iteration of Kodaira-Spencer map starting from $L$ as the initial line bundle one obtains non-zero map

$$
L \otimes \operatorname{ker}\left(\theta^{\otimes(*)}\right)^{\vee} \rightarrow S^{m} \Omega_{\bar{Y}}(\log \bar{S}) .
$$

As the sheaf on the left-hand side is a tensor product of a generically ample line bundle with a non-negative sheaf and the image is generically ample and $\Omega_{\bar{Y}}^{1}(\log \bar{S})$ is semi-positive, the bigness of $\omega_{Y}(\bar{S})$ follows.

\section{Bigness of $\Omega_{\bar{Y}}^{1}(\log \bar{S})$ for a Log Base $(\bar{Y}, \bar{S})$ Parametrizing Varieties with Semi-ample Canonical Line Bundles}

The following construction has been introduced in [29,30]. Let $f: V \rightarrow U$ be a smooth family of polarized manifolds of dimension $n$ with semi-ample canonical line bundle and with maximal variation. We take a good partial compactification $f: X \rightarrow Y$ of the original family $f: V \rightarrow U$, which satisfies:

- $\quad X$ and $Y$ are quasi-projective manifolds, $U \subset Y$ and $V \subset X$. The map $f$ restricted to $V$ coincides with $f: V \rightarrow U$.

- $\quad S:=Y \backslash U$ is smooth and $\Delta:=f^{*}(S)$ is relatively normal crossing over $S$. Hence, $f: X \rightarrow Y$ is a $\log$ smooth projective morphism between the $\log$ pairs $(X, \Delta)$ and $(Y, S)$.

- $\quad Y$ has a smooth projective compactification $\bar{Y}$ such that $\bar{Y} \backslash U=: \bar{S}$ is a normal crossing divisor and $\operatorname{codim}(\bar{Y} \backslash Y) \geq 2$.

\subsection{Deformation Higgs Bundle (Sheaf) $\left(F_{0}, \tau_{0}\right)$ Arising from Kodaira-Spencer Map}

We start with the classical Kodaira-Spencer map on the $\log$ smooth family $f: X \rightarrow Y$ :

$$
T_{Y}(-\log S) \stackrel{\tau_{0}^{n, 0}}{\longrightarrow} R^{1} f_{*} T_{X / Y}(-\log \Delta),
$$

or equivalently

$$
\mathcal{O}_{Y} \stackrel{\tau_{0}^{n, 0}}{\longrightarrow} R^{1} f_{*} T_{X / Y}(-\log \Delta) \otimes \Omega_{Y}^{1}(\log S) .
$$

Tensoring the Kodaira-Spencer map $\tau_{0}^{n, 0}$ with $R^{q} f_{*} T_{X / Y}^{q}(-\log \Delta)$ and then composing with the wedge product $\wedge$, we define the extended Kodaira-Spencer maps $\tau_{0}^{p, q}$ as the composition:

$$
\begin{aligned}
T_{Y}(-\log S) \otimes R^{q} f_{*} T_{X / Y}^{q}(-\log \Delta) \stackrel{\tau_{0}^{n, 0} \otimes \mathrm{Id}}{\longrightarrow} & R^{1} f_{*} T_{X / Y}(-\log \Delta) \otimes R^{q} f_{*} T_{X / Y}^{q}(-\log \Delta) \\
& \stackrel{\wedge}{\rightarrow} R^{q+1} f_{*} T_{X / Y}^{q+1}(-\log \Delta) .
\end{aligned}
$$

Denote $F_{0}^{p, q}=R^{q} f_{*} T_{X / Y}^{q}(-\log \Delta)$. Putting all terms together we obtain the so-called deformation Higgs bundle (sheaf) attached to $f: X \rightarrow Y$ :

$$
\left(F_{0}, \tau_{0}\right):=\left(\bigoplus_{p+q=n} F_{0}^{p, q}, \bigoplus_{p+q=n} \tau_{0}^{p, q}\right) .
$$


One checks that the sum of the extended Kodaira-Spencer maps $\tau_{0}=\oplus \tau_{0}^{p, q}$ satisfies the integrability condition $\tau_{0} \wedge \tau_{0}=0$ using the associativity and anti-commutivity of the cup product on Dolbeault cohomology of the tangent sheaf. By taking the reflexive hull $F^{p, q}$ of $F_{0}^{p, q}$ on $\bar{Y}$, the Higgs field

$$
\tau_{0}^{p, q}: \quad F_{0}^{p, q} \rightarrow F_{0}^{p-1, q+1} \otimes \Omega_{Y}^{1}(\log S)
$$

extends to a Higgs field

$$
\tau^{p, q}: \quad F^{p, q} \rightarrow F^{p-1, q+1} \otimes \Omega_{\bar{Y}}^{1}(\log \bar{S})
$$

on $\bar{Y}$, since $\operatorname{codim}(\bar{Y} \backslash Y) \geq 2$. So we obtain a Higgs bundle (sheaf) $(F, \tau)$ on $\bar{Y}$ as an extension of the Kodaira-Spencer map of $f: X \rightarrow Y$.

We note that for a family of Calabi-Yau $n$-folds the Higgs bundle $(F, \tau)$ is nothing but the graded Higgs bundle of the VHS associated to the middle cohomology of $f$ after tensoring with the Hodge line bundle $f_{*} \Omega_{X / Y}^{n}(\log \Delta)^{\vee}$.

Motivated by the negativity of the kernel of the Higgs map arising from PVHS, we would like to compare $(F, \tau)$ with a Higgs bundle arising from the geometric origin and show the negativity of the kernel of Kodaira-Spencer map $\tau$.

Via linear algebra

$$
F_{0}^{p, q}=R^{q} f_{*}\left(\Omega_{X / Y}^{p}(\log \Delta) \otimes \mathcal{L}^{-1}\right) / \bmod \text { torsions }
$$

with $p+q=n$ and $\mathcal{L}=\Omega_{X / Y}^{n}(\log \Delta)$ we see that there is a close relation between $(F, \tau)$ and the graded Higgs bundle $(E, \theta)=\bigoplus R^{q} f_{*}\left(\Omega_{X / Y}^{p}(\log \Delta), \theta\right)$ arising from PVHS of the middle cohomology of $f$. Indeed, if $\mathcal{L}$ has a non-zero section, then it induces a natural Higgs sheaf map from $(F, \tau)$ into $(E, \theta)$. In Section 3.5 we will see that this idea can be realized once some positive power of $\mathcal{L}$ has non-zero sections.

\subsection{Comparing $(F, \tau)$ with Higgs Bundle Arising from the Geometry Origin}

Given an ample line bundle $A$ on $\bar{Y}$ there are two versions of the comparison map.

Version 1. Replacing $f: X \rightarrow Y$ by taking a self-fibre product to a higher power By taking a self fibre product $f^{(n)}: \bar{X}^{(n)} \rightarrow \bar{Y}$ to a higher power and a cyclic cover $\pi: \bar{Z} \rightarrow$ $\bar{X}^{(n)}$ we obtain a Higgs sheaf map

$$
\rho: \quad\left(F_{0}^{(n)}, \tau_{0}^{(n)}\right) \rightarrow(E, \theta) \otimes A^{-1},
$$

where $\left(F_{0}^{(n)}, \tau_{0}^{(n)}\right)$ denote the deformation Higgs bundle associated to $f^{(n)}: X^{(n)} \rightarrow Y$ and $(E, \theta)$ is the logarithmic graded Higgs bundle on $\bar{Y}$ of the quasi-canonical extension of the PVHS of the middle cohomology of the induced family $g: Z \stackrel{\pi}{\rightarrow} X^{(n)} \stackrel{f^{(n)}}{\longrightarrow} Y$ with the normal crossing degeneration locus $\bar{S}+\bar{T}$ (after a blowing up of $\bar{Y}$ of the degeneration locus of $g$ ).

Version 2. Replacing $Y$ by a Kawamata cover $Y^{\prime} \rightarrow Y$ to raise the power of $A$ By taking a Kawamata cover $\psi: Y^{\prime} \rightarrow Y$ and a cyclic cover $\pi: Z \rightarrow X^{\prime}$ of the fibre product $f^{\prime}: X^{\prime} \rightarrow Y^{\prime}$ induced by the base change, we obtain a Higgs sheaf map

$$
\rho: \quad \psi^{*}(F, \tau) \rightarrow(E, \theta) \otimes \psi^{*} A^{-1},
$$


where $(E, \theta)$ is the logarithmic graded Higgs bundle of the quasi-canonical extension of PVHS of the middle cohomology of the induced family $g: Z \stackrel{\pi}{\rightarrow} X^{\prime} \stackrel{f^{\prime}}{\rightarrow} Y^{\prime}$.

We emphasize the following crucial property of the comparison map. Although the Higgs field $\theta$ on $E$ has in general bigger singularity $\bar{S}+\bar{T}$, its restriction to $\rho\left(F^{(n)}\right)$ has only singularity on the original degeneration locus $\bar{S}$. We will briefly describe the construction of $(E, \theta)$ in Section 3.5 .

\subsection{Negativity of the Kernel of Kodaira-Spencer Map}

The Higgs sheaf map $\rho$ is a sort of Torelli map, which relates the negativity of the kernel of $\theta$ to the negativity of the kernel of $\tau$. First we discuss the injectivity of $\rho$.

Proposition 2 We take $(F, \tau)$ either as the deformation Higgs bundle associated to a higher power selffibre product of the original family or as the pulled back of the deformation Higgs bundle associated to the original family via a Kawamata base change. Writing $\rho=\bigoplus \rho^{p, q}$,

(1) $\rho^{n, 0}: O_{\bar{Y}}=F^{n, 0} \rightarrow E^{n, 0} \otimes A^{-1}$ is injective.

(2) If the canonical line bundle of the fibres is ample, then every $\rho^{p, q}$ is injective at all points of $U \backslash T$.

(3) If the canonical line bundle of the fibres is big, then $\rho^{n-1,1}$ is injective at all points of $U \backslash T$.

(4) If the fibres admit good minimal models, then the following composition map

$$
T_{\bar{Y}}(\log \bar{S}) \otimes F^{n, 0} \stackrel{\rho^{n, 0}}{\longrightarrow} T_{\bar{Y}}(-\log \bar{S}) \otimes \rho^{n, 0}\left(F^{n, 0}\right) \stackrel{\theta^{n, 0}}{\longrightarrow} E^{n-1,1} \otimes A^{-1}
$$

is injective at every point of a Zariski open subset of $U \backslash T$.

The injectivity in (1) is tautological. The injectivity in (2) respectively in (3) follows from Kodaira type respectively Bogomolov-Sommese vanishing theorem [29]. The comparison map in (4) is similar (in every way) to the Kodaira-Spencer map on a family of CalabiYau $n$-folds over $U$. Viehweg and Zuo show the map is non-zero and Deng [5] shows it is injective at generic point. Both arguments are global and crucially rely on the seminegativity of $\operatorname{ker}\left(\theta^{n, 0}\right)$ and the strictly negativity of $A^{-1}$. The injectivity of $\theta^{n, 0} \circ \rho^{n, 0}$ in (4) is weaker than the injectivity in (3), but strong enough to show all hyperbolicities on $U$ except for Kobayashi hyperbolicity.

As a consequence of the the non-triviality of the comparison map, we get the negativity of the kernel of Kodaira-Spencer map. To illustrate the idea we assume that the canonical line bundle along the fibres are ample (in general one works with the image $\rho(F, \tau) \subset$ $(E, \theta) \otimes A^{-1}$ and pays the attention to the property that the restriction of $\theta$ to $\rho(F)$ has only singularities in $\bar{S}$ and

$$
\theta^{n, 0}: \quad T_{\bar{Y}}(-\log \bar{S}) \rightarrow E^{n-1,1} \otimes A^{-1}
$$

is injective at the generic point). We have an embedding of Higgs bundles

$$
(F, \tau) \subset(E, \theta) \otimes A^{-1}
$$

and

$$
\operatorname{ker}(\tau) \subset \operatorname{ker}(\theta) \otimes A^{-1} .
$$

Since $(E, \theta)$ is the quasi-canonical extension of a polarized VHS on $U \subset \bar{Y}=U \cup \bar{S} \cup \bar{T}$, Griffiths curvature formula for $\operatorname{ker}(\theta)$ yields the semi-negativity on $\operatorname{ker}(\theta)$; more precisely, 
the Hodge metric on $\operatorname{ker}(\theta)$ outside $S+T$ extends to a degenerated metric on $\bar{Y}$ with seminegative curvature in the sense of currents. Hence, we see that $\operatorname{ker}(\tau)$ is negative on $\bar{Y}$ with the following consequences.

\section{Proposition 3}

1. Under the comparison map $\rho$, the image of the kernel of the Kodaira-Spencer map of a family of varieties with semi-ample canonical line bundle along the fibres (in general admitting good minimal model) and of maximal variation is strictly negative.

2. Taking the maximal non-zero iteration of Kodaira-Spencer map on $(F, \tau)$ for the case the canonical line bundle on fibres is ample (for semi-ample case take the image $\rho(F, \tau) \subset(E, \theta))$

$$
S^{l} T_{\bar{Y}}(-\log S) \stackrel{\tau^{l} \neq 0}{\longrightarrow} \operatorname{ker}\left(\tau^{n-l, l}\right) \subset \operatorname{ker}\left(\theta^{n-l, l}\right) \otimes A^{-1}
$$

its dual gives rise to a generically ample subsheaf

$$
\mathcal{A}:=\operatorname{im}\left(A \otimes \operatorname{ker}\left(\theta^{n-l, l}\right)^{\vee}\right) \stackrel{\tau^{l \vee} \neq 0}{\longrightarrow} S^{l} \Omega_{\bar{Y}}^{1}(\log \bar{S}),
$$

i.e. $\Omega_{\bar{Y}}^{1}(\log \bar{S})$ is big.

3. Assume the canonical line bundle on the fibres is ample. If $(G, \tau) \subset(F, \tau)$ be a subHiggs bundle, then $\operatorname{det} G^{\vee}$ is big. In particular, $\left(\bigoplus_{j=l}^{n} R^{j} f_{*} T_{X / Y}^{j}(-\log \Delta), \tau\right)$ is a sub-Higgs bundle of $(F, \tau)$ for any $0 \leq l \leq n$. Hence $\bigotimes_{j=l}^{n} \operatorname{det}\left(R^{j} f_{*} T_{X / Y}^{j}(-\log \Delta)\right)^{\vee}$ is big.

Proof 3. Take the second version of the comparison map

$$
\rho: \quad \psi^{*}(G, \tau) \rightarrow(E, \theta) \otimes \psi^{*} A^{-1},
$$

where $\rho$ is injective. Let $r:=\operatorname{rank} G$, then the sub-Higgs line bundle

$$
\psi^{*} \operatorname{det}(G, \tau) \rightarrow \wedge^{r}(E, \theta) \otimes \psi^{*} A^{-r}
$$

lies in the kernel of the Higgs field on the right-hand side, as a graded Higgs line bundle has vanishing Higgs field. This shows that $\phi^{*} \operatorname{det} G^{\vee}$ is big on $\bar{Y}^{\prime}$ and hence $\operatorname{det} G^{\vee}$ is big on $\bar{Y}$.

Remark 1 It would be interesting to compare the bigness of the line bundle

$$
\bigotimes_{j=l}^{n} \operatorname{det}\left(R^{j} f_{*} T_{X / Y}^{j}(-\log \Delta)\right)^{\vee}
$$

with the bigness of the Griffiths augmented line bundle from PVHS.

\subsection{Pseudo-effectivity of $\Omega_{\bar{Y}}^{1}(\log \bar{S})^{\otimes N}$ and Various Hyperbolicities of $U$}

Campana and Paun [4] proved that $\Omega_{\bar{Y}}(\log \bar{S})$ is pseudo-effective. Namely, for any quotient sheaf $Q$ of $\Omega_{\bar{Y}}(\log \bar{S})^{\otimes N}$ and for any movable curve $\bar{C} \subset \bar{Y}$ one has $c_{1}(Q) \bar{C} \geq 0$. The proof relies on the existence of a generically ample subsheaf $\mathcal{A} \subset S^{l} \Omega_{\bar{Y}}^{1}(\log \bar{S})$. Again combining with this ample subsheaf $\mathcal{A}$ they showed the $\log$ pair $(\bar{Y}, \bar{S})$ is of $\log$ general type, the so-called conjecture on Viehweg hyperbolicity. 
The maximal non-zero iteration of Kodaira-Spencer map on the image Higgs bundle $\rho((F, \tau) \otimes A)$

$$
S^{l} T_{\bar{Y}}(-\log S) \otimes A \stackrel{\rho(\tau)^{l} \neq 0}{\longrightarrow} \operatorname{ker}\left(\rho \tau^{n-l, l}\right) \subset \operatorname{ker}\left(\theta^{n-l, l}\right)
$$

defines a complex Finsler pseudometric (a further modification of the Fubini-Study metric on $A$ is eventually needed [30] and [21]) such that the holomorphic sectional curvature is strictly negative. All hyperbolicities on $U$ except the Kobayashi hyperbolicity follows from this metric. In particular, very recently Deng et al. [7] have shown the big Picard theorem holds true in $U$. As for Kobayashi hyperbolicity, a further modification by taking the Finsler metric as the sum of iterations of all lengths by To and Yeung [26] (also see [5]) is needed for constructing a non-degenerated and strictly negatively curved Finsler metric.

The following proposition shall give a Hodge theoretical counterpart and interpretation of the strict negativity of the holomorphic sectional curvature of the modified Finisler metric defined by taking the sum of iterations of all lengths.

Proposition 4 The determinant of the image Higgs sheaf by taking iterations of KodairaSpencer map of all lengths

$$
\left(A \oplus T_{Y}(\log S) \otimes A \oplus S^{2} T_{Y}(-\log S) \otimes A \oplus \cdots \oplus S^{l} T_{Y}(-\log S) \otimes A\right) \rightarrow(E, \theta)
$$

is non-positive.

Comments 1 The pseudo-effectivity of $\Omega_{\bar{Y}}^{1}(\log S)$ together with the big subsheaf $\mathcal{A}$ in the symmetric power of $\Omega_{\bar{Y}}^{1}(\log S)$ makes the $\log$ pair $(\bar{Y}, \bar{S})$ similar to a base space admitting a locally injective Torelli map. For more applications we would like to ask for the positivity of $\log$ differential forms pulled back to subvarieties. More precisely, regarding the second component of the deformation Higgs bundle $F^{n-1,1}=R^{1} f_{*} T_{X / Y}(-\log S)$ as the pulled back of the log tangent sheaf on the moduli space via the moduli map one would like to know if any type non-positivity of $F^{n-1,1}$ restricted to any subvariety holds true? In a recent paper [15] Javanpeykar, Sun and Zuo used the argument by taking the sum of iterations of Kodaira-Spencer map specifically on an invertible subsheaf in the pulled back $\phi^{*} F^{n-1,1}$ via a $\log$ map $\phi$ into $(Y, S)$ and showed that

Proposition 5 [15] Let $\phi:\left(C, S_{C}\right) \rightarrow(Y, S)$ be a non constant map from a log curve $(C, S)$, then any invertible subsheaf $L \subset \phi^{*} F^{n-1,1}$ one has

$$
\operatorname{deg} L<\frac{(n-1) \operatorname{deg} \Omega_{C}^{1}\left(\log S_{C}\right)}{2} .
$$

Consequently, Javanpeykar, Sun and Zuo proved a new version of the Shafarevich conjecture, the so-called finiteness of pointed families of polarized varieties.

Theorem 1 (Weak-pointed Shafarevich conjecture [15]) Let $k$ be an algebraically closed field of characteristic zero, let $f: V \rightarrow U$ be a smooth family of h-dimensional varieties with semi-ample canonical line bundle and such that the moduli map is quasi finite. Then $U$ has the following finiteness property: If $N \geq \frac{1}{2}(h-1)(2 g(\bar{C})-2+\#(\bar{C} \backslash C))$ is an integer, $u_{1}, \ldots, u_{N} \in U(k)$ are points, $C$ is a smooth quasi projective connected curve over $k$, and $c_{1}, \ldots, c_{N} \in C(k)$ are pairwise distinct points, then the set of non-constant morphisms $\phi: C \rightarrow U$ with $\phi\left(c_{1}\right)=u_{1}, \ldots, \phi\left(c_{N}\right)=u_{N}$ is finite. 
Conjecture 1 The degree of any subsheaf of the pullback $\phi^{*} F^{n-1,1}$ via any non-constant $\log \operatorname{map} \phi:\left(C, S_{C}\right) \rightarrow(Y, S)$ is non-positive.

Conjecture 1 should have more stronger consequences on the geometry of $(Y, S)$. For example, it implies the one pointed Shafarevich Conjecture asked in [15].

So far we have discussed various notions of hyperbolicity in the sense of complex analysis, to end this section we like to pose a conjecture on the bigness of the fundamental group of $U$.

One notes that a punctured Riemann surface $U$ is hyperbolic if and only if $\pi_{1}(U)$ is infinite and nonabelian.

Definition 3 (Milnor [19]) A growth function $\ell$ associated to a finitely generated group $G$ is defined as follows: For each positive integer $s$ let $\ell(s)$ be the number of distinct group elements which can be expressed as words of length $\leq s$ with a fixed choice of generators and their inverses.

Theorem 2 (Milnor [19]) The fundamental group of a compact Riemannian manifold $M$ with all Riemannian sectional curvatures less than zero has exponential growth, i.e.

$$
\ell(s) \geq a^{s}
$$

for some $a>1$.

The proof relies on Günther's volume comparison theorem on the exponential growth of the volume of the geodesic ball on the universal cover $\tilde{M}$. We make

Conjecture 2 Let $U$ be a base parameterizing polarized manifolds with semi-ample line bundle and of maximal variation. Then $\pi_{1}(U)$ grows at least exponentially.

The idea supporting Conjecture 2 goes back to a question asked by Jürgen Jost many years ago. A horizontal subvariety in a period domain is locally embedded into the associated real symmetric space under the natural projection. As the real symmetric space has non-positive Riemannian sectional curvature, Jost asked if the Hodge metric, as a Kähler metric on the horizontal subvariety enjoys the same type of property for the Riemannian sectional curvature. However, Conjecture 1 asks more, via the comparison map and the maximal non-zero iteration of the Kodaira-Spencer map one hopes that the negativity of the Riemannian sectional curvature on the real symmetric space can be further "transformed" into the degenerated Finsler metric on $U$ discussed here. Similar to the approach in proving the complex hyperbolicity on $U$ we hope to construct a Riemann-Finsler metric on $U$ via the iteration of Kodaira-Spencer map, whose curvature has certain negativity. In our situation the complex Finsler metric naturally induces a Riemann-Finsler metric $d s_{\mathbb{R} F i n}^{2}$ on $U$. We are aware that in general the Riemannian curvature decreasing principle does not hold true for real sub-manifolds. Very recently together with Steven Lu and Ruiran Sun we observed the fact that pluriharmonicity of the composition of the horizontal period map with the projection to the symmetric space of non-compact type implies decreasing Riemannian curvature still holds true in a weak form. We expect this weak form of the negative sectional curvatures can be used to show Günter's volume comparison inequality and get the solution of Conjecture 2 by Milnor's original argument. 


\subsection{Constructing the Comparison Map in Section 3.2, a Hodge Theoretic Interpretation}

The main issue in the comparison map is that we would like to give the relative Čech cohomology of log tangent sheaf $T_{X / Y}^{p}(-\log \Delta)$ a Hodge theoretical interpretation. Hence, the negativity (or positivity) results from Hodge theory can be applied. The construction is the gluing of the fiberwise construction, which goes back to the works of Esnault-Viehweg and Kawamata.

\subsubsection{Cyclic (Kummer) Cover in the Absolute Case}

Let $X$ be a smooth projective variety. Cyclic covers of $X$ are classical and were invented mainly to prove Kodaira type vanishing theorems for the Čech cohomology of differential forms (twisted with an anti big line bundle) of type $p+q<\operatorname{dim} X$, see [8] for details. Note that for the comparison map we focus on the cohomology of type $p+q=\operatorname{dim} X$.

Let $\mathcal{L}$ be a line bundle on $X$ with $H^{0}\left(X, \mathcal{L}^{v}\right) \neq 0$ for some positive integer $v$. Then any non-zero section $s \in H^{0}\left(X, \mathcal{L}^{v}\right)$ will induce naturally a comparison map

$$
\rho_{s}: \quad H^{q}\left(X, \Omega_{X}^{p} \otimes \mathcal{L}^{-1}\right) \rightarrow H^{q}\left(Z, \Omega_{Z}^{p}\right),
$$

where $Z$ stands for the $v$-th cyclic (Kummer) cover of $s$, see [8, Section 3].

Assume that the zero divisor $\operatorname{div}(s)=: D$ is smooth. The $v$-th root out of $s$ defines a smooth cyclic cover $\pi: Z \rightarrow X$ ramified on $D \subset X$. The Galois group of the cover $(\mathbb{Z} / \nu \mathbb{Z})$ acts on $Z$ and on $\pi_{*}\left(\Omega_{Z}^{p}\right)$. The eigenspace decomposition reads as:

$$
\pi_{*}\left(\Omega_{Z}^{p}\right)=\Omega_{X}^{p} \oplus \bigoplus_{i=1}^{\nu-1} \Omega_{X}^{p}(\log D) \otimes \mathcal{L}^{-i}
$$

which induces an isomorphism

$$
H^{q}\left(Z, \Omega_{Z}^{p}\right) \simeq H^{q}\left(X, \pi_{*} \Omega^{p} Z\right)=H^{q}\left(X, \Omega_{X}^{p}\right) \oplus \bigoplus_{i=1}^{\nu-1} H^{q}\left(X, \Omega_{X}^{p}(\log D) \otimes \mathcal{L}^{-i}\right) .
$$

Picking up the 1 st eigenspace $\Omega_{X}^{p}(\log D) \otimes \mathcal{L}^{-1}$, the inclusion of sheaves

$$
\Omega_{X}^{p} \otimes \mathcal{L}^{-1} \hookrightarrow \Omega_{X}^{p}(\log D) \otimes \mathcal{L}^{-1}
$$

induces a map

$$
\rho^{p, q}: \quad H^{q}\left(X, \Omega_{X}^{p} \otimes \mathcal{L}^{-1}\right) \rightarrow H^{q}\left(X, \Omega_{X}^{p}(\log D) \otimes \mathcal{L}^{-1}\right) \subset H^{q}\left(Z, \Omega_{Z}^{p}\right) .
$$

In general, $D$ could be singular and have some components with multiplicities. We first take a blowing up $\delta: W \rightarrow X$ to make $\delta^{*}(D)=: B=\sum_{j} \alpha_{j} B_{j}$ being normal crossing. Then we choose a suitable Kummer cover, the so-called Kawamata cover, $\pi: Z \rightarrow W$ by adding the $v$-th root of $\pi^{*} s$ and the $v$-th roots of some sections from additional line bundles such that $Z$ is smooth and the ramification locus is a normal crossing divisor containing the divisor $\sum_{j: \frac{\alpha_{j}}{v} \notin \mathbb{N}} B_{j}=: B^{\prime}$. Again, by applying the eigenspace decomposition and the inclusion of sheaves of $\log$ differential forms one obtains

$$
H^{q}\left(X, \Omega_{X}^{p} \otimes \mathcal{L}^{-1}\right) \rightarrow H^{q}\left(W, \Omega_{W}^{p}\left(\log B^{\prime}\right) \otimes\left(\sigma^{*} \mathcal{L}\right)^{(-1)}\right) \subset H^{q}\left(Z, \Omega_{Z}^{p}\right),
$$

where $\left(\delta^{*} \mathcal{L}\right)^{(-1)}$ is slightly large than $\delta^{*} \mathcal{L}^{-1}$, see [8] for details. 
- Logarithmic rank one local system $\left(\mathcal{L}^{-1}, \nabla\right)$ on $X$ arising from the cyclic cover $\pi: Z \rightarrow X$. In fact, the eigenspace associated to 1

$$
H^{q}\left(Z, \Omega_{Z}^{p}\right)_{1}=H^{q}\left(X, \Omega_{X}^{p}(\log D) \otimes \mathcal{L}^{-1}\right)
$$

can be directly interpreted as Hodge cohomology of a filtered logarithmic de Rham bundle on the log pair $(X, D)$ as follows, see [8, Sections 2 and 3]. The direct image $f_{*}\left(O_{Z}, d\right)$ of the trivial de Rham bundle on $Z$ is again a de Rham bundle on $X$ with logarithmic singularities along $D$. The 1-eigenspace of $f_{*}\left(\mathcal{O}_{Z}\right)$ for the Galois action is $\mathcal{L}^{-1}$ together with the integrable connection $\nabla$ coming from $d$ with $\log$ pole along $D$ of the finite local monodromy along $D$ of the order $v$. The de Rham complex with the coefficient $\left(\mathcal{L}^{-1}, \nabla\right)$ has the form

$$
0 \rightarrow \mathcal{L}^{-1} \stackrel{\nabla}{\rightarrow} \Omega_{X}^{1}(\log D) \otimes \mathcal{L}^{-1} \stackrel{\nabla}{\rightarrow} \cdots \stackrel{\nabla}{\rightarrow} \Omega_{X}^{n}(\log D) \otimes \mathcal{L}^{-1} \rightarrow 0,
$$

which has Hodge-to-de Rham $E_{1}$-degeneration w.r.t. the truncated de Rham subcomplexes using twisted harmonic forms defined by a singular locally constant metric below. One checks that the Hodge cohomology of this de Rham complex is $H^{q}\left(X, \Omega_{X}^{p}(\log D) \otimes \mathcal{L}^{-1}\right)$.

- Singular locally constant metric on $\left(\mathcal{L}^{-1}, \nabla\right)$. The constant metric on $\mathcal{O}_{Z}$ descends to a locally constant metric on $\pi_{*}\left(\mathcal{O}_{Z}, d\right)$ which has singularities exactly along the ramification divisor $D \subset X$ of $\pi: Z \rightarrow X$. Hence, it restricts to a locally constant metric on the eigenspace $\left(\mathcal{L}^{-1}, \nabla\right)$. More explicitly, let $t$ be a local coordinate function on an analytic open subset $V$ vanishing on $D \cap V$ then $t^{\frac{1}{v}}$ is a local base of the eigen sub line bundle $\mathcal{L}^{-1} \subset \pi_{*} \mathcal{O}_{Z}$ on $V$. The constant metric on $\mathcal{O}_{Z}$ defines a locally constant metric $h$ on $\mathcal{L}^{-1}$ with the local expression $|t|^{\frac{1}{v}}$. One uses this locally constant metric to define harmonic representatives for the Betti cohomology of $\mathbb{L}^{-1}$, similar to the constant local system $\mathbb{C}$ and to prove $E_{1}$-degeneration directly on $X$. This is mentioned in the book [8].

\subsubsection{Cyclic (Kummer) Cover for a Family $f: X \rightarrow Y$ in the Relative Case and the Comparison Map}

- Comparison map by taking self-fibre product. Let $f: X \rightarrow Y$ be a good partial compactification of a smooth family $f: V \rightarrow U$ of fibre dimension $n$ with maximal variation and such that the canonical line bundle along the fibres are semi-ample. Recall that for $\mathcal{L}:=\Omega_{X / Y}^{n}(\log \Delta)$, Kawamata and Viehweg showed that $f_{*} \omega_{X / Y}^{v}$ is big for $v \gg 0[17$, $27,28]$. By a slight modification Viehweg-Zuo showed that $f_{*}\left(\mathcal{L}^{\nu}\right)$ is globally generated over a Zariski open subset $U^{\prime} \subset U$ and for $\nu \gg 0$. Fix an ample line bundle $A$ on $\bar{Y}$. Then $\mathcal{L}^{v} \otimes A^{-1}$ is again globally generated over $f^{-1}\left(U^{\prime}\right)$ for $v \gg 0$. By replacing the original family by the self fibre product of a suitable power $f^{(r)}: X^{(r)} \rightarrow Y$ ([29, proof of 1.4 iii), p. 27]) or by a Kawamata base change $\phi: Y^{\prime} \rightarrow Y$ ([29, proof of 1.4 iv), p. 27]) we may assume $\mathcal{L}^{v} \otimes f^{*}\left(A^{-v}\right)$ is globally generated over $f^{-1}\left(U^{\prime}\right)$.

Choose a generic non-zero section $s$ of $\mathcal{L}^{v} \otimes f^{*}\left(A^{-v}\right)$ such that the zero divisor $D:=\operatorname{div}(s)$ intersects a general fibre of $f$ smoothly. Let $T \subset Y$ denote the closure of the discriminant of the map $H \cap V \rightarrow U$, which is the locus where the intersection $D_{y}:=D \cap f^{-1}(y)$ becomes singular. Leaving out some codimension two subschemes, we may assume that $S+T$ is a smooth divisor. Let $\Sigma:=f^{*}(T)$ and we keep the notation $\Delta=f^{*}(S)$. We take a blowing up $\delta: W \rightarrow X$ with centers in $D+\Delta+\Sigma$ and such that 
$\delta^{*}(D+\Delta+\Sigma)$ is normal crossing and the composition map

$$
h: \quad W \stackrel{\delta}{\rightarrow} X \stackrel{f}{\rightarrow} Y
$$

is $\log$ smooth as a morphism between $\log$ pairs

$$
h:\left(W, \delta^{*}(D+\Delta+\Sigma)\right) \rightarrow(Y,(S+T)) .
$$

We write $\mathcal{M}:=\delta^{*}\left(\mathcal{L} \otimes f^{*} A^{-1}\right)$ and $B:=\delta^{*}(D)$, then $\mathcal{M}^{v}=O_{W}(B)$. One takes the $v$-th cyclic cover of the divisor $B$ and choose $Z$ to be a desingularization of this covering and obtains the induced new family

$$
g: Z \stackrel{\pi}{\rightarrow} X \stackrel{f}{\rightarrow} Y .
$$

Write $\Pi=g^{-1}(S+T)$. Then the restriction $g: Z_{0}=Z \backslash \Pi \rightarrow Y \backslash(S+T)$ is a smooth family. We may assume that $\bar{S}+\bar{T}$ is normal crossing in $\bar{Y}$. The quasi-canonical extension of the filtered de Rham bundle of the locally constant system $R^{n} f_{*}\left(\mathbb{C}_{Z_{0}}\right)$ gives rise to a locally free filtered logarithmic de Rham bundle

$$
\nabla: \mathcal{V} \rightarrow \mathcal{V} \otimes \Omega_{\bar{Y}}(\log (\bar{S}+\bar{T}))
$$

and the graded Higgs bundle

$$
(E, \theta):=\left(\bigoplus_{q=0}^{k} E^{k-q, q}, \bigoplus_{q=0}^{k} \theta^{k-q, q}\right) .
$$

The Galois group of $g$ acts on the local system hence on the Higgs bundle. Let $(E, \theta)_{1}$ denote 1st eigen sub Higgs bundle.

Remark 2 Similar to Section 3.5.1 the line bundle $\mathcal{M}^{-1}$ underlies a structure of de Rham bundle $\left(\mathcal{M}^{-1}, \nabla\right)$ on $W$ with logarithmic singulartiy on $\delta^{*}(D+\Delta+\Sigma)$. Let $\mathbb{M}^{-1}$ denote the local system. Then $(E, \theta)_{1}$ is the graded Higgs bundle of the quasi-canonical extension of the filtered de Rham bundle of the variation of mixed Hodge structures of the local system $R^{n} h_{*}\left(\mathbb{M}_{W \backslash \delta^{*}(D+\Delta+\Sigma)}^{-1}\right)$ on $Y \backslash(S+T)$ and with logarithmic pole on $(S+T)$.

We now make the comparison between the deformation Higgs bundle $(F, \tau)$ for $f$ : $X \rightarrow Y$ with $(E, \theta)_{1} \otimes A^{-1}$. Just remember that the cyclic cover $\pi: Z \rightarrow W$ is defined by taking the $\nu$-root out of the divisor $\delta^{*} D \in\left|\left(\delta^{*} \mathcal{L} \otimes h^{*} A^{-1}\right)^{v}\right|$. Therefore $(E, \theta)_{1} \otimes A^{-1}$ can be computed by taking higher direct image $R^{\bullet} h_{*}$ of the tautological exact sequence of the log smooth map

$$
h:(W, \delta(D+\Delta+\Sigma)) \rightarrow(Y,(S+T))
$$

twisted by $\delta^{*} \mathcal{L}^{-1}$, that is

(I) $\begin{aligned} & 0 \rightarrow h^{*} \Omega_{Y}^{1}(\log (S+T)) \otimes \Omega_{W / Y}^{p-1}\left(\log \delta^{*}(D+\Delta+\Sigma)\right) \otimes \delta^{*} \mathcal{L}^{-1} \\ & \rightarrow \operatorname{Gr}\left(\Omega_{W}^{p}(\log (\delta(D+\Delta+\Sigma))) \otimes \delta^{*} \mathcal{L}^{-1} \rightarrow \Omega_{W / Y}^{p}(\log (\delta(D+\Delta+\Sigma))) \otimes \delta^{*} \mathcal{L}^{-1} \rightarrow 0 .\right.\end{aligned}$

On the other hand, the Higgs bundle $\left(F_{0}, \tau\right)$ with $F_{0}^{n-q, q}=R^{q} f_{*}\left(\Omega_{X / Y}^{n-q}(\log \Delta) \otimes \mathcal{L}^{-1}\right)$ can be computed by taking higher direct images $R^{\bullet} f_{*}$ of the tautological exact sequence of the $\log$ smooth map $f:(X, \Delta) \rightarrow(Y, S)$ twisted with $\mathcal{L}^{-1}$

(II) $0 \rightarrow f^{*} \Omega_{Y}^{1}(\log S) \otimes \Omega_{X / Y}^{p-1}(\log \Delta) \otimes \mathcal{L}^{-1} \rightarrow \operatorname{Gr}\left(\Omega_{X}^{p}(\log \Delta)\right) \otimes \mathcal{L}^{-1}$

$\rightarrow \Omega_{X / Y}^{p}(\log \Delta) \otimes \mathcal{L}^{-1} \rightarrow 0$, 
where

$$
\operatorname{Gr}\left(\Omega_{X}^{p}(\log \Delta)\right)=\Omega_{X}^{p}(\log \Delta) / f^{*} \Omega_{Y}^{2}(\log S) \otimes \Omega_{X}^{p-2}(\log \Delta) .
$$

The Hurwitz formula for the pullback of logarithmic differential forms via $\delta: W \rightarrow X$ induces an inclusion of the short exact sequence $\delta^{*}(I I) \hookrightarrow(I)$, which induces a map between higher direct images $\rho^{p, q}: F_{0}^{p, q} \rightarrow E_{0}^{p, q}$ and commuting with the edge morphisms.

- Comparison map by taking Kawamata cover. The construction here is similar to the previous case. Fixing an ample line bundle then $\mathcal{L}^{v} \otimes A^{-1}$ is big for $v \gg 0$. Instead of taking self fibre product to higher power we take a Kawamata cover $\psi: Y^{\prime} \rightarrow Y$ so that $\psi^{*} A=A^{\prime v}$ for an ample line bundle on $Y^{\prime}$. Let $f^{\prime}: X^{\prime} \rightarrow Y^{\prime}$ denote the fibre product of the base change and $\mathcal{L}^{\prime}=f_{*}^{\prime} \Omega_{X^{\prime} / Y^{\prime}}^{n}\left(\log \Delta^{\prime}\right)$ then $\left(\mathcal{L}^{\prime} \otimes A^{\prime-1}\right)^{v} \simeq \psi^{*}\left(\mathcal{L}^{v} \otimes A^{-1}\right)$ is big. By taking the $v$-th cyclic cover $\pi^{\prime}: Z^{\prime} \rightarrow X^{\prime}$ of a section $s$ of $\left(\Omega_{X^{\prime} / Y^{\prime}}^{n}\left(\log \Delta^{\prime}\right) \otimes f^{\prime *} A^{\prime-1}\right)^{\nu}$ we get the comparison map by the same type of the construction as before.

\section{Length of a Family, Characteristic Varieties and Distributions on a Base}

\subsection{Introducing the Notion of the Length of a Family and a Criterion for the Rigidity}

Given a family $f: X \rightarrow Y$ of varieties of dimension $n$ with of semi-ample canonical line bundle and such that the moduli map into the moduli space is quasi finite. Recall the $i$-th iteration of the Kodaira-Spencer map

$$
\tau^{i}: \mathcal{O}_{Y} \simeq F^{n, 0} \stackrel{\tau^{n, 0}}{\longrightarrow} F^{n-1,1} \otimes \Omega_{Y}^{1}(\log S) \rightarrow \cdots \stackrel{\tau^{n-(i-1), i-1}}{\longrightarrow} F^{n-i, i} \otimes S^{i} \Omega_{Y}^{1}(\log S) .
$$

For $i=n$ (dimension of the fibres) one obtains a coupling

$$
\tau^{n}: \mathcal{O}_{Y} \rightarrow F^{0, n} \otimes S^{n} \Omega_{Y}^{1}(\log S) .
$$

If $f$ is a family of Calabi-Yau manifolds or in general replacing the deformation Higgs bundle by the graded Higgs bundle of the VHS of the middle cohomology of the family then $\tau^{n}$ coincides with the Yukawa coupling or in general Griffiths-Yukawa coupling.

Definition 4 We introduce the length of the iteration of Kodaira-Spencer map of the family $f: X \rightarrow Y$ to be

$$
\zeta(f)=\operatorname{Min}\left\{i \geq 1 \mid \tau^{i}=0\right\}-1 .
$$

For a comparison map of the second version

$$
\rho: \psi_{\rho}^{*}\left((F, \tau) \otimes A_{\rho}\right) \rightarrow(E, \theta)_{\rho}
$$

via a Kawamata cover $\psi_{\rho}: Y_{\rho}^{\prime} \rightarrow Y$ we take the maximal non-zero iteration of the KodairaSpencer map valued in $\psi^{*} T_{Y}(\log S)$ on the image Higgs sheaf

$$
\rho\left(\psi_{\rho}^{*}\left((F, \tau) \otimes A_{\rho}\right)\right) \subset(E, \theta)_{\rho}
$$


and obtain a generically ample subsheaf in $\psi_{\rho}^{*} S^{\zeta(\rho)} \Omega_{Y}^{1}(\log S)$,

$$
\mathcal{A}_{\rho} \hookrightarrow \psi_{\rho}^{*} S^{\zeta(\rho)} \Omega_{Y}^{1}(\log S),
$$

where $\zeta(\rho)$ is called the length of the iteration under a comparison map $\rho$ of the second version. It is clear that $\zeta(\rho) \leq \zeta(f)$ and $\operatorname{rank} \mathcal{A}_{\rho} \leq h^{\zeta(\rho)}\left(X_{y}, T_{X_{y}}^{\zeta(\rho)}\right)$.

\subsubsection{Non-rigid Families and Products Base}

Given a $\log$ pair $\left(Z, S_{Z}\right)$, the $\log$ morphisms $\phi:\left(Z, S_{Z}\right) \rightarrow(Y, S)$, (i.e. $\phi\left(Z \backslash S_{Z}\right) \subset U$ and we consider generically finite $\log$ morphisms) are parameterized by a scheme $\mathcal{H}:=$ $\operatorname{Hom}\left(\left(Z, S_{Z}\right),(Y, S)\right)$ of locally finite type. By the boundedness theorem [18], the scheme $\mathcal{H}$ is of finite type. A morphism $\phi_{0}$ is called rigid if $\phi$ is an isolated point in $\mathcal{H}$. If $\phi_{0}$ is not rigid then there is exists a positive dimensional $\log$ pair $\left(T, S_{T}\right)$ such that $\phi_{0}$ extends to a generically finite morphism

$$
\phi:\left(Z, S_{Z}\right) \times\left(T, S_{T}\right) \rightarrow(Y, S)
$$

by removing a codimension-2 subscheme. In general, we are interested in a product $\prod_{i}\left(Z_{i}, S_{i}\right)$ into $(Y, S)$ via a generically finite map.

Via the comparison map the length $\zeta(f)$ provides an upper bound of the number of log subvarieties appearing as factors in a product in $(Y, S)$.

Proposition 6 (Viehweg-Zuo [29]) If there exists a generically finite map from a product $\prod_{i=1}^{m}\left(Z_{i}, S_{i}\right)$ of $m$ log pairs into $(Y, S)$ then $m \leq \zeta(f) \leq \operatorname{dim} f^{-1}$.

This proposition suggests that $\zeta(f)$ looks like the rank of a locally bounded symmetric space. Indeed, applying Proposition 6 to the universal family of polarized abelian $g$-folds one shows that the maximal number of subvarieties in a product in $\overline{\mathcal{A}}_{g}$ is equal to $g$. It is well-known that there exists products of $g$ copies of modular curves as the so-called polydisc embedding in $\overline{\mathcal{A}}_{g}$ parameterizing abelian varieties isogeny to products of $g$-copies of elliptic curves. Here $g$ is defined as the rank of the group $\operatorname{Sp}(2 g, \mathbb{R})$.

\section{Proposition 7}

1. If there exists a generically finite morphism from a product $\prod_{i=1}^{\zeta(f)}\left(Z_{i}, S_{i}\right)$ of the maximal $\zeta(f) \log$ pairs into $(Y, S)$ then all $\Omega_{Z_{i}}^{1}\left(\log S_{i}\right)$ are generically ample and $\operatorname{dim} Z_{i} \leq h^{\zeta(f)}\left(X_{y}, T_{X_{y}}^{\zeta(f)}\right)$.

2. (A criterion for rigidity for families of algebraic surfaces in terms of the second geometric genus of the fibre surfaces.) Let $f: X \rightarrow Y$ be a family of algebraic surfaces with semi-ample canonical line bundle and such that the moduli map from the base to the moduli space is quasi-finite then any generically finite morphism $\phi_{0}:(Z, S) \rightarrow(Y, S)$ is rigid if $\operatorname{dim} Z>h^{0}\left(X_{y}, \omega_{X_{y}}^{\otimes 2}\right)$.

Proof 1 . The pullback of $\mathcal{A} \hookrightarrow S^{\zeta(f)} \Omega_{Y}^{1}(\log S)$ to $\prod_{i=1}^{\zeta(f)}\left(Z_{i}, S_{i}\right)$ has the form

$$
\mathcal{A} \hookrightarrow \Omega_{Z_{1}}^{1}\left(\log S_{1}\right) \otimes \cdots \otimes \Omega_{Z_{\zeta(f)}}^{1}\left(\log S_{\zeta(f)}\right)
$$

(see the proof for Corollary 6.4 in [29] and to be more precise we shall work on the Kawamata cover $\psi_{\rho}: Y^{\prime} \rightarrow Y$ ). Restricting the above map, for example, to the factor $Z_{1}$ passing 
through the generic point, then $\mathcal{A}_{Z_{1}}$ is generically ample and from the inclusion map

$$
\begin{aligned}
\mathcal{A}_{Z_{1}} & \left.\hookrightarrow\left(\Omega_{Z_{1}}^{1}\left(\log S_{Z_{1}}\right) \otimes \Omega_{Z_{2}}^{1}\left(\log S_{Z_{2}}\right) \otimes \cdots \otimes \Omega_{Z_{\zeta(f)}}^{1}\left(\log S_{Z_{\zeta(f)}}\right)\right)\right|_{Z_{1}} \\
& \simeq \Omega_{Z_{1}}^{1}\left(\log S_{Z_{1}}\right)^{\oplus \prod_{i=2}^{\zeta(f)} \operatorname{dim} Z_{i}} .
\end{aligned}
$$

We obtain a non-zero map

$$
\alpha: \mathcal{A}_{Z_{1}} \rightarrow \Omega_{Z_{1}}^{1}\left(\log S_{Z_{1}}\right)
$$

Claim $1 \alpha$ is generically surjective.

Proof of Claim 1 Consider the non-zero sub sheaf $\alpha\left(\mathcal{A}_{Z_{i}}\right) \hookrightarrow \Omega_{Z_{1}}^{1}\left(\log S_{Z_{1}}\right)$ and let $d:=$ $\operatorname{rank} \alpha\left(\mathcal{A}_{Z_{i}}\right)$ then $d \leq \operatorname{rank} \Omega_{Z_{1}}^{1}\left(\log S_{Z_{1}}\right)=\operatorname{dim} Z_{1}$. One observes that the invertible sub sheaf $\operatorname{det} \alpha\left(\mathcal{A}_{Z_{i}}\right) \hookrightarrow \Omega_{Z_{1}}^{d}\left(\log S_{Z_{1}}\right)$ is big. By Bogomolov's lemma ([1, 12.2. Theorem 4], here we use the version for logarithmic forms) any invertible sub sheaf of $\Omega_{Z_{1}}^{d}\left(\log S_{Z_{1}}\right)$ has Kodaira-dimension less then or equal to $d$. Hence, the bigness of $\operatorname{det} \alpha\left(\mathcal{A}_{Z_{i}}\right)$ implies $d=\operatorname{dim} Z_{1}$. Claim 1 is done.

By Claim 1 we obtain a generically surjective map $\mathcal{A}_{Z_{1}} \rightarrow \Omega_{Z_{i}}^{1}\left(\log S_{i}\right)$. In particular,

$$
\operatorname{dim} Z_{i} \leq \operatorname{rank} \mathcal{A}_{Z_{1}} \leq h^{\zeta(f)}\left(X_{y}, T_{X_{y}}^{\zeta(f)}\right)
$$

2. For such a family $f: X \rightarrow Y$ of surfaces we have then the rank $\zeta(f)=1$ or 2 . For $\zeta(f)=1$, then the map $\mathcal{A} \rightarrow \Omega_{Y}^{1}(\log S)$ is surjective. Since $\mathcal{A}$ is generically ample, $\Omega_{Y}^{1}(\log S)$ is generically ample. Hence, any generically finite morphism $\phi_{0}:\left(Z, S_{Z}\right) \rightarrow$ $(Y, S)$ is rigid.

For $\zeta(f)=2$. Given a non-rigid generically finite morphism $\phi_{0}:\left(Z, S_{Z}\right) \rightarrow(Y, S)$, we must show that $\operatorname{dim} Z \leq h^{0}\left(X_{y}, \omega^{2}\right)$. By 4.1.1, there exists a log curve $\left(T, S_{T}\right)$ such that on the complement $\left(W, S_{W}\right)$ of a codim-2 sub scheme $\Sigma$ of $\left(Z, S_{Z}\right) \times\left(T, S_{T}\right)$ the morphism $\phi_{0}$ extends to a generically finite morphism

$$
\phi:\left(W, S_{W}\right) \rightarrow(Y, S) .
$$

We denote $f_{W}: X_{W} \rightarrow W$ the pullback family of $f: X \rightarrow Y$ via $\phi:\left(W, S_{W}\right) \rightarrow(Y, S)$.

Claim 2 The length $\zeta\left(f_{W}\right)$ of the family $f_{W}$ is equal to 2 .

Proof of Claim 2 If $\zeta\left(f_{W}\right)=1$, then the maximal non-zero iteration of Kodaria-Spncer map of the family $f_{W}$ has length 1 and induces a non-zero map

$$
\tau: \mathbb{A}_{W} \rightarrow \Omega_{W}^{1}\left(\log S_{W}\right),
$$

where $\mathbb{A}_{W}$ is generically ample. Noting that $\left(W, S_{W}\right)$ is the complement of a codimension-2 sub scheme in $\left(Z, S_{Z}\right) \times\left(T, S_{T}\right)$ we obtain an extension

$$
\tau: \mathbb{A}_{Z \times T} \rightarrow \Omega_{Z \times T}^{1}\left(\log S_{Z \times T}\right) .
$$

By Bogomolov's lemma $\tau$ is generically surjective. Hence, $\Omega_{Z \times T}^{1}\left(\log S_{Z \times T}\right)$ is generically ample. But, on the other hand $\Omega_{Z \times T}^{1}\left(\log S_{Z \times T}\right)$ can not be generically ample. Claim 2 is completed.

Since $\zeta\left(f_{W}\right)=2$ we obtain a non-zero map

$$
\begin{aligned}
\tau^{2}: S^{2} \Omega_{Z}^{1}\left(\log S_{Z}\right) \rightarrow & S^{2} \Omega_{Z \times T}^{1}\left(\log S_{Z \times T}\right) \\
& =S^{2} \Omega_{Z}^{1}\left(\log S_{Z}\right) \boxplus S^{2} \Omega_{T}^{1}\left(\log S_{T}\right) \boxplus \Omega_{Z}^{1}\left(\log S_{Z}\right) \otimes \Omega_{T}^{1}\left(\log S_{T}\right) .
\end{aligned}
$$


Since $\mathcal{A}_{Z \times T}$ is generically ample, the restriction of $S^{2} \Omega_{Z}^{1}\left(\log S_{Z}\right)$ to the $T$-factor and the restriction of $S^{2} \Omega_{T}^{1}\left(\log S_{T}\right)$ to the $Z$-factor are trivial sheaves, therefor the map $\tau^{2}$ factors through

$$
\tau^{2}: \mathcal{A}_{Z \times T} \rightarrow \Omega_{Z}^{1}\left(\log S_{Z}\right) \otimes \Omega_{T}^{1}\left(\log S_{T}\right) .
$$

Applying Bogomolov lemma to the restriction of $\tau^{2}$ to a generic $Z$-factor

$$
\mathcal{A}_{Z} \rightarrow \Omega_{Z}^{1}\left(\log S_{Z}\right)
$$

shows that $\operatorname{rank} \Omega_{Z}^{1}\left(\log S_{Z}\right) \leq \operatorname{rank} \mathcal{A}_{Z} \leq h^{0}\left(X_{y}, \omega^{2}\right)$.

Assume $f: X \rightarrow Y$ in Proposition 7 is a family of Calabi-Yau manifolds or hypersurfaces in $\mathbb{P}^{N}$ of degree $d \geq N+1$ [31] shows a much more stronger result:

Proposition 8 Let $f: V \rightarrow U=U_{1} \times \cdots \times U_{l}$ be a smooth family of Calabi-Yau $m$-folds or a normalized family of hypersurfaces in $\mathbb{P}^{N}$ of degree $d \geq N+1$ (admitting a particle good compactification $f: X \rightarrow Y$ with $Y=Y_{1} \times \cdots \times Y_{l}$ removed a codimension-2 sub scheme) and let the corresponding moduli map be generically finite. Then the natural map induced by $k$-th iteration of the Kodaira-Spencer map on the deformation Higgs bundle

$$
\bigoplus_{1 \leq i_{1}<\cdots<i_{k} \leq l} p_{i_{1}}^{*} T_{U_{i_{1}}} \otimes \cdots \otimes p_{i_{k}}^{*} T_{U_{i_{k}}} \rightarrow R^{k} f_{*} T_{V / U}^{k}
$$

is injective. In particular,

$$
\sum_{1 \leq i_{1}<\cdots<i_{k} \leq l} \operatorname{dim} U_{i_{1}} \cdots \operatorname{dim} U_{i_{k}} \leq h^{k}\left(V_{y}, T_{V_{y}}^{k}\right) .
$$

Propositions 6, 7 and 8 support the following conjecture.

Conjecture 3 ([31, Conjecture 3.2]) Proposition 8 holds true for any family of varieties with semi-ample canonical line bundle.

\subsection{Characteristic Varieties Attached to a Family}

We would like to introduce a finer infinitesimal invariant to characterize products in $(Y, S)$. In the study of the metric rigidity problem on a locally bounded symmetric domain $\mathcal{D}$, N. Mok [20] has introduced the so-called characteristic bundles $\left\{S_{i}\right\}$ in the projective tangent bundle of $\mathcal{D}$ using a maximal set of strongly orthogonal positive non-compact roots in a Cartan subalgebra $h$ of the Lie algebra of the Lie group of Hermitian type. Note that $S^{1}$ consists of those tangents with minimal holomorphic sectional curvature. Motivated by Mok's work Sheng-Zuo [23] have introduced the notion of characteristic varieties for PVHS of Calabi-Yau type using iterations of Kodaira-Spencer map on the graded Higgs bundle. For the case of universal families of abelian varieties over Shimura varieties, the characteristic varieties coincides with Mok's characteristic bundles over Shimura varieties. Sheng, Xu and Zuo [24] and Robles [22] have found further applications in characterization of Gross's canonical variations of Hodge structure of Calabi-Yau type over bounded symmetric tube domains.

Motivated by the above construction, we introduce characteristic varieties attached to a family $f: X \rightarrow Y$ of $n$-dim varieties with semi-ample canonical line bundle along the fibres and with the maximal variation as follows. 
Assume the canonical line bundle along the fibres is ample: by taking $k$-th iterated Kodaira-Spencer map on the deformation Higgs bundle

$$
S^{k} T_{Y}(-\log S) \stackrel{\tau^{k}}{\rightarrow} F^{n-k, k}, \quad 1 \leq k \leq \zeta(f)
$$

we define the $k$-th characteristic variety $S^{k} \subset \mathbb{P}\left(T_{Y}(-\log S)\right)$ fibrewise by

$$
S_{y}^{k}=\left\{\left.v \in T_{Y}(-\log S)\right|_{y}: \tau^{k+1}\left(v^{\otimes(k+1)}\right)=0\right\} .
$$

For the case the canonical line bundle is semi-ample along the fibres we perform this construction via a comparison map over a Kawamata cover. In this way we obtain an increasing filtration of subvarieties in the projective bundle of log tangent bundle over $Y$

$$
\emptyset=S^{0} \subset S^{1} \subset \cdots \subset S^{k} \subset S^{k+1} \subset \cdots \subset S^{\zeta(f)-1} \subset S^{\zeta(f)}=\mathbb{P}\left(T_{Y}(-\log S)\right) .
$$

The characteristic varieties provide an infinitesimal characterization of products of subvarieties in $(Y, S)$ in the following sense.

Proposition 9 If there is a generically finite map $\phi$ from the $\log$ pair $\prod_{i=1}^{l}\left(Z_{i}, S_{i}\right)$ into $(Y, S)$ then each factor $Z_{i}$ lies in leaves of the distribution defined by $S^{\zeta(f)-l+1}$, i.e.

$$
\left.\mathbb{P}\left(T_{Z_{i}}\left(-\log S_{i}\right)\right) \subset S^{\zeta(f)-l+1}\right|_{Z_{i}} .
$$

Proof We take the map restricted to the factor the $\prod_{i=1}^{l-1}\left(Z_{i}, S_{i}\right)$, then the family pulled back to $\prod_{i=1}^{l-1}\left(Z_{i}, S_{i}\right)$ is not rigid. By Corollary 6.5 in [29]

$$
\left.\mathbb{P}\left(T_{\prod_{i=1}^{l-1} Z_{i}}\left(-\log S_{\prod_{i=1}^{l-1} Z_{i}}\right)\right) \subset S^{\zeta(f)-d_{1}}\right|_{\prod_{i=1}^{l-1} Z_{i}}
$$

and

$$
\left.\mathbb{P}\left(T_{\prod_{i=1}^{l-1} Z_{i}}\left(-\log S_{\prod_{i=1}^{l-1} Z_{i}}\right)\right) \not \subset S^{\zeta(f)-d_{1}-1}\right|_{\prod_{i=1}^{l-1} Z_{i}}
$$

for some $d_{1} \geq 1$. Repeating the argument for further $(l-2)$ times we finish the proof.

Motivated by the above proposition we introduce the notion on special sub varieties in $(Y, S)$.

Definition 5 A $\log$ subvariety $(Z, S)$ of $(Y, S)$ is called special and of degree $l$ if it lies in leaves of the distribution defined by $S^{\zeta(f)-l}$, i.e.

$$
\left.\mathbb{P}\left(T_{Z}(-\log S)\right) \subset S^{\zeta(f)-l}\right|_{Z} \quad \text { and }\left.\quad \mathbb{P}\left(T_{Z}(-\log S)\right) \not \subset S^{\zeta(f)-l-1}\right|_{Z} .
$$

Conjecture 4 Given a family $f: X \rightarrow Y_{1} \times \cdots \times Y_{l}$ of canonically polarized varieties over a product base. Then the family is dominated by a finite map from the product of $l$ families $f_{i}: X_{i} \rightarrow Y_{i}, 1 \leq i \leq l$.

Faltings [9] found examples of families of abelian varieties such that Conjecture 4 does not hold true. However, the VHS attached to those families does decompose over a number field. If the canonical line bundle of the fibres is ample one hopes the decomposition of the base induces a decomposition on the fibers. 
Acknowledgements This note grows up on several letter exchanges with Phillip Griffiths. I would like to thank him for his interest, his suggestions for the exposition, and in particular for inspired discussions concerning the notion of the length of a family. I would also like to thank Steven Lu for discussions on various notions of bigness of vector bundles, Ruiran Sun for discussions on the integrability of the KodairaSpencer map and Raju Krishnamoorthy for pointing out several unclear points in the note. Finally, I would like to thank three referees' valuable comments and suggestions on this note.

Funding Open Access funding enabled and organized by Projekt DEAL.

Open Access This article is licensed under a Creative Commons Attribution 4.0 International License, which permits use, sharing, adaptation, distribution and reproduction in any medium or format, as long as you give appropriate credit to the original author(s) and the source, provide a link to the Creative Commons licence, and indicate if changes were made. The images or other third party material in this article are included in the article's Creative Commons licence, unless indicated otherwise in a credit line to the material. If material is not included in the article's Creative Commons licence and your intended use is not permitted by statutory regulation or exceeds the permitted use, you will need to obtain permission directly from the copyright holder. To view a copy of this licence, visit http://creativecommons.org/licenses/by/4.0/.

\section{References}

1. Bogomolov, F.A.: Holomorphic tensors and vector bundles on projective varieties. Math USSR Izv. 13, 499 (1979)

2. Brunebarbe, Y., Klingler, B., Totaro, B.: Symmetric differentials and the fundamental group. Duke Math. J. 162, 2797-2813 (2013)

3. Cattani, E., Kaplan, A., Schmid, W.: Degeneration of Hodge structures. Ann. Math. (2) 123, 457-535 (1986)

4. Campana, F., Păun, M.: Orbifold generic semi-positivity: an application to families of canonically polarized manifolds. Ann. Inst. Fourier (Grenoble) 65, 835-861 (2015)

5. Deng, Y.: Pseudo Kobayashi hyperbolicity of base spaces of families of minimal projective manifolds with maximal variation. arXiv:1809.05891. JEMA (to appear) (2018)

6. Deng, Y.: Big Picard theorem and algebraic hyperbolicity for varieties admitting a variation of Hodge structures. arXiv:2001.04426 (2020)

7. Deng, Y., Lu, S., Sun, R.-R., Zuo, K.: Big Picard theorems for moduli spaces of polarized varieties. arXiv:1911.02973 (2019)

8. Esnault, H., Viehweg, E.: Lectures on Vanishing Theorems DMV Seminar, vol. 20. Birkhäuser, Basel (1992)

9. Faltings, G.: Arakelov's theorem for Abelian varieties. Invent. math. 73, 337-348 (1983)

10. Green, M., Griffiths, P.A.: Positivity of vector bundles and Hodge theory. arXiv: 1803.07405 (2018)

11. Griffiths, P.A.: Periods of integrals on algebraic manifolds. I. Construction and properties of the modular varieties. Amer. J. Math. 90, 568-626 (1968)

12. Griffiths, P.A.: Periods of integrals on algebraic manifolds. II. Local study of the period mapping. Amer. J. Math. 90, 805-865 (1968)

13. Griffiths, P.A.: Periods of integrals on algebraic manifolds. III. Some global differential-geometric properties of the period mapping. Inst. Hautes Etudes Sci. Publ. Math. 38, 125-180 (1970)

14. Griffiths, P.A., Schmid, W.: Locally homogeneous complex manifolds. Acta Math. 123, 253-302 (1969)

15. Javanpeykar, A., Sun, R.R., Zuo, K.: The Shafarevich conjecture revisited: Finiteness of pointed families of polarized varieties. arXiv:2005.05933 (2020)

16. Jost, J., Yau, S.-T.: Harmonic mappings and algebraic varieties over function fields. Amer. J. Math. 115, 1197-1227 (1993)

17. Kawamata, Y.: Minimal models and the Kodaira dimension of algebraic fibre spaces. J. Reine Angew. Math. 363, 1-46 (1985)

18. Kovács, S.J., Lieblich, M.: Boundedness of families of canonically polarized manifolds: A higher dimensional analogue of Shafarevich conjecture. Ann. Math. (2) 172, 1719-1748 (2011)

19. Milnor, J.: A note on curvature and fundamental group. J. Differ. Geom. 2, 1-7 (1968)

20. Mok, N.: Metric Rigidity Theorems on Hermitian Locally Symmetric Manifolds. Series in Pure Mathematics, vol. 6. World Scientific, Singapore (1989) 
21. Popa, M., Taji, B., Wu, L.: Brody hyperbolicity of base spaces of certain families of varieties. Algebra Number Theory 13, 2205-2242 (2019)

22. Robles, C.: Characterization of Calabi-Yau variations of Hodge structures over tube domains by characteristic forms. Math. Ann. 371, 1229-1253 (2018)

23. Sheng, M., Zuo, K.: Polarized variation of Hodge structures of Calabi-Yau type and characteristic subvarieties over bounded symmetric domains. Math. Ann. 348, 211-236 (2010)

24. Sheng, M., Xu, J.X., Zuo, K.: The monodromy groups of Dolgachev's CY moduli spaces are Zariski dense. Adv. Math. 272, 699-742 (2015)

25. Simpson, C.: Constructing variations of Hodge structures using Yang-Mills theory and applications to uniformization. J. Amer. Math. Soc. 1, 867-918 (1988)

26. To, W.-K., Yeung, S.-K.: Finsler metrics and Kobayashi hyperbolicity of the moduli spaces of canonically polarized manifolds. Ann. Math. (2) 181, 547-586 (2015)

27. Viehweg, E.: Weak positivity and the additivity of the Kodaira dimension for certain fibre spaces. In: Iitaka, S. (ed.) Algebraic Varieties and Analytic Varieties. Advanced Studies in Pure Mathematics, vol. 1, pp. 329-353. North-Holland Publ. (1983)

28. Viehweg, E.: Weak positivity and the additivity of the Kodaira dimension for certain fibre spaces II. The local Torelli map. In: Ueno, K. (ed.) Classification of Algebraic and Analytic Manifolds, Progress in Mathematics, vol. 39, pp. 567-589. Birkhäuser, Boston (1983)

29. Viehweg, E., Zuo, K.: Base spaces of non-isotrivial families of smooth minimal models. In: Bauer, I., et al. (eds.) Complex Geometry (Göttingen, 2000), pp. 279-328. Springer, Berlin (2002)

30. Viehweg, E., Zuo, K.: On the Brody hyperbolicity of moduli spaces for canonically polarized manifolds. Duke Math. J. 118, 103-150 (2003)

31. Viehweg, E., Zuo, K.: Complex multiplication, Griffiths-Yukawa couplings, and rigidity for families of hypersurfaces. J. Algebraic Geom. 14, 481-528 (2005)

32. Zuo, K.: On the negativity of kernels of Kodaira-Spencer maps on Hodge bundles and applications. Asian J. Math. 4, 279-301 (2000)

Publisher's Note Springer Nature remains neutral with regard to jurisdictional claims in published maps and institutional affiliations. 